\title{
Communication and Creative Thinking in Agile Software Development
}

\author{
Broderick Crawford ${ }^{1,2}$, Claudio León de la Barra ${ }^{1}$, and Patricio Letelier ${ }^{3}$ \\ ${ }^{1}$ Pontificia Universidad Católica de Valparaíso, Chile, cleond@ucv.cl \\ ${ }^{2}$ Universidad Técnica Federico Santa María, Chile, broderick.crawford@ucv.cl \\ ${ }^{3}$ Universidad Politécnica de Valencia, España, letelier@dsic.upv.es
}

\begin{abstract}
This paper describes and analyses how a eXtreme Programming (XP) team work can use some ideas from Psychology and Computer Science fostering creativity and innovation in Software Development. The roles for creative team in order to have a chance for creative thinking, communication, innovation, collaboration and knowledge sharing are addressed to Agile Software Development teams.
\end{abstract}

Keywords: Creativity, Software Development, Agile Methodologies, eXtreme Programming.

\section{Introduction}

Psychology and Computer Science are growing in a interdisciplinary relationship mainly because human and social factors are very important in developing software and hardware. Software is developed for people and by people [1], but surprisingly, most of software engineering research is technical and deemphasizes the human and social aspects. By other hand, the traditional development process of new products that is a fundamental part in marketing has been recently criticized by Kotler and Trías de Bes [2]. They point out that fundamental creative aspects are not considered at all and as a consequence this development is not useful, viable or innovative. In this context, it is interesting to consider the new proposals of agile methodologies for software development in order to analyze and evaluate them at the light of the existing creative expositions, mainly considering the teamwork practices.

The agile principles and values have emphasized the importance of communication, collaboration and interaction in the software development and, by other hand, creative work commonly involves collaboration in some form and it can be understood as an interaction between an individual and a socio-cultural context. In relation with the joint work between users and agile software 
developers, there are very interesting cases in the work of Jeff Sutherland, the inventor of the popular Scrum Agile Development Process [3-7]. The most notorious agile methods: Scrum and eXtreme Programming XP [8], had attained worldwide fame for its ability to increase the productivity of software teams by several magnitudes through empowering individuals, fostering a team-oriented environment, and focusing on project transparency and results [9-13].

We believe that the innovation and development of new products is an interdisciplinary issue [14], we are interested in the study of the potential of new concepts and techniques to foster creativity in software engineering [15]. This paper is organized as follows: in section 2 we explain the motivation of this work fixing the relevance of Creativity in Software Development. Section 3 is about central aspects in Creativity. Section 4 gives a brief overview of XP and its phases and roles. In Section 5 we show how the XP Creative Team Can Operate. Section 6 presents a comparison between roles in creative teams and roles in XP teams. Finally, in Section 7 we conclude the paper.

\section{Creative Thinking in Software Development}

Software engineering is a knowledge intensive process, this underlies the need for communication, collaboration and knowledge sharing support to share domain expertise between the customer and the development team [16]. Since human creativity is thought as the source to resolve complex problem or create innovative products, one possibility to improve the software development process is to design a process which can stimulate the creativity of the developers. There are few studies reported on the importance of creativity in software development. In management and business, researchers have done much work about creativity and obtained evidence that the employees who had appropriate creativity characteristics, worked on complex, challenging jobs, and were supervised in a supportive, non-controlling fashion, produced more creative work. Then, according to the previous ideas the use of creativity in software development is undeniable, but requirements engineering is not recognized as a creative process in all the cases [17]. In a few publications the importance of creativity has been investigated in all the phases of software development process [18,15,19] and mostly focused in the requirements engineering [20-22]. Nevertheless, the use of techniques to foster creativity in requirements engineering is still shortly investigated, it is not surprising that the role of communication and interaction is central in many of the creativity techniques. The importance of creative thinking is expected to increase over the next decade [23]. In [20, 24] very interesting open questions are proposed: Is inventing part of the requirements activity? It is if we want to advance. So who does the inventing? We cannot rely on the customer to know what to invent. The designer sees his task as deriving the optimal solution to the stated requirements. We can not rely on programmers because they are far 
away from the work of client to understand what needs to be invented. Requirements analysts are ideally placed to innovate. They understand the business problem, have updated knowledge of the technology, will be blamed if the new product does not please the customer, and know if inventions are appropriate to the work being studied. In short, requirements analysts are the people whose skills and position allows, indeed encourages, creativity. In [25] the author, a leading authority on cognitive creativity, identifies basic types of creative processes: exploratory creativity explores a possible solution space and discovers new ideas, combinatorial creativity combines two or more ideas that already exist to create new ideas, and transformational creativity changes the solution space to make impossible things possible. Then, most requirements engineering activities are exploratory, acquiring and discovering requirements and knowledge about the problem domain. Requirements engineering practitioners have explicitly focused on combinatorial and transformational creativity. In relation with the active participation of the users in software development, Holzinger has very valuable work making usability practitioners first-class citizens in the process [26-29].

\section{Creativity in the Development of New Products}

The creativity definitions are numerous [30-32], therefore, considering the object of analysis in the present paper: a software development teamwork responding to the requirements of a specific client for a particular problem, a suitable definition is the one raised by Welsch [33]: Creativity is the process of generating unique products by transformation of existing products. These products, tangible and intangible, must be unique only to the creator, and must meet the criteria of purpose and value established by the creator.

More specifically, and from an eminently creative perspective, it is possible to distinguish three aspects at the interior of a group developing new products (purposes, performance and structure):

a) The purposes that the team tries to reach, which demand two scopes of results [34-38]:

- Those related to the creative result that must be original, elaborated, productive and flexible.

- Those related to the creative team, so that it reaches its goals, developing cognitive abilities and presenting an improved disposition to the change. All this in order to obtain a better creative team performance in the future.

b) The performance shown by the team in connection with the main aspects of the complex dynamics that the persons build inside a team. We describe three aspects: 
- The personal conditions of the members of the team, in terms of the styles and cognitive abilities, the personality, their intrinsic motivation and knowledge [32,39,30,34].

- The organizational conditions in which the creative team is inserted, and that determines, at least partly, its functioning. These conditions, in the extent that pre-sent/display certain necessary particular characteristics although non sufficient- for the creative performance. They emphasize in special the culture (communication, collaboration, trust, conflict handle, pressure and learning) [32,40,41]; the internal structure (formalization, autonomy and evaluation of the performance) [32,40,41,39]; the team available resources (time disposition) $[32,40,30]$ and the physical atmosphere of work [31].

- The conditions of performance of the creative team, mainly the creative process realized, which supposes the set of specific phases that allow assure the obtaining of a concrete result (creative product) [31, 42].

c) The structure of the creative team, particularly the group characteristics, such as norms, cohesiveness, size, diversity, roles, task and problem-solving approaches [32].

Of the mentioned aspects, we deepen in those referred to the structure and performance of the team for the development of new products, specially considering: the creative process and the roles surrounding this process.

\subsection{The Phases of the Creative Process}

The creative process constitutes the central aspect of team performance, because it supposes a serie of clearly distinguishable phases that had to be realized by one or more of the team members in order to obtain a concrete creative result. Considering the creativity as a "nonlinear" process some adjustments are necessary including feedbacks whose "destiny" can be anyone of the previous phases. The phases, on the basis of Wallas [42] and Leonard and Swap [31], are the following ones:

- Initial preparation: the creativity will bloom when the mental ground is deep, fertile and it has a suitable preparation. Thus, the deep and relevant knowledge, and the experience precedes the creative expression.

- Encounter: the findings corresponding to the perception of a problematic situation. For this situation a solution does not exist. It is a new problem.

- Final preparation: it corresponds to the understanding and foundation of the problem. It's the immersion in the problem and the use of knowledge and analytical abilities. It includes search for data and the detailed analysis of factors and variables.

- Generation of options: referred to produce a menu of possible alternatives. It supposes the divergent thinking. It includes, on one hand, finding principles, lines or addresses, when making associations and 
uniting different marks of references and, on the other hand, to generate possible solutions, combinations and interpretations.

- Incubation: it corresponds to the required time to reflect about the elaborated alternatives, and "to test them mentally".

- Options Choice: it corresponds to the final evaluation and selection of the options. It supposes the convergent thinking.

- Persuasion: closing of the creative process and communication to other persons.

\subsection{The Roles in a Creative Team}

Lumsdaine and Lumsdaine [43] raise the subject of the required cognitive abilities (mindsets) for creative problem resolution. Their typology is excellent for the creative team, and the different roles to consider. These roles are the following ones:

- Detective. In charge of collecting the greatest quantity of information related to the problem. It has to collect data not making judgements even when it thinks that it has already understood the problem exactly.

- Explorer. Detects what can happen in the area of the problem and its context. It thinks on its long term effects and it anticipates certain developments that can affect the context (in this case, the team). The explorer perceives the problem in a broad sense.

- Artist. Creates new things, transforming the information. It must be able to break his schemes to generate eccentric ideas, with imagination and feeling.

- Engineer. Is the one in charge of evaluating new ideas. It must make converge the ideas, in order to clarify the concepts and to obtain practical ideas that can be implemented for the resolution of problems.

- Judge. Must do a hierarchy of ideas and decide which of them will be implemented (and as well, which ones must be discarded). Additionally, it must detect possible faults or inconsistencies, as well as raise the corresponding solutions. Its role must be critical and impartial, having to look for the best idea, evaluating the associated risks.

- $\quad$ Producer. In charge of implementing the chosen ideas.

Leonard and Swap [31] have mentioned additional roles, possible to be integrated with the previous ones, because they try to make more fruitful the divergence and the convergence in the creative process:

- The provoker who takes the members of the team "to break" habitual mental and procedural schemes to allow the mentioned divergence (in the case of the "artist") or even a better convergence (in the case of the "engineer"). 
- Think tank that it is invited to the team sessions to give a renewed vision of the problem-situation based on his/her expertise and experience.

- The facilitator whose function consists in helping and supporting the team work in its creative task in different stages.

- The manager who cares for the performance and specially for the results of the creative team trying to adjust them to the criteria and rules of the organization (use of resources, due dates).

Kelley and Littman [44], on the other hand, have raised a role typology similar to Lumsdaine and Lumsdaine [43], being interesting that they group the roles in three categories: those directed to the learning of the creative team (susceptible of corresponding with the detective, explorer, artist, provoker and think tank roles); others directed to the internal organization and success of the team (similar to the judge, facilitator and manager roles); and roles whose purpose is to construct the innovation (possibly related to the role of the engineer and judge).

\section{Agile Software Development with eXtreme Programming}

Extreme Programming is an iterative approach to software development [8], the process is shown in Figure 1.

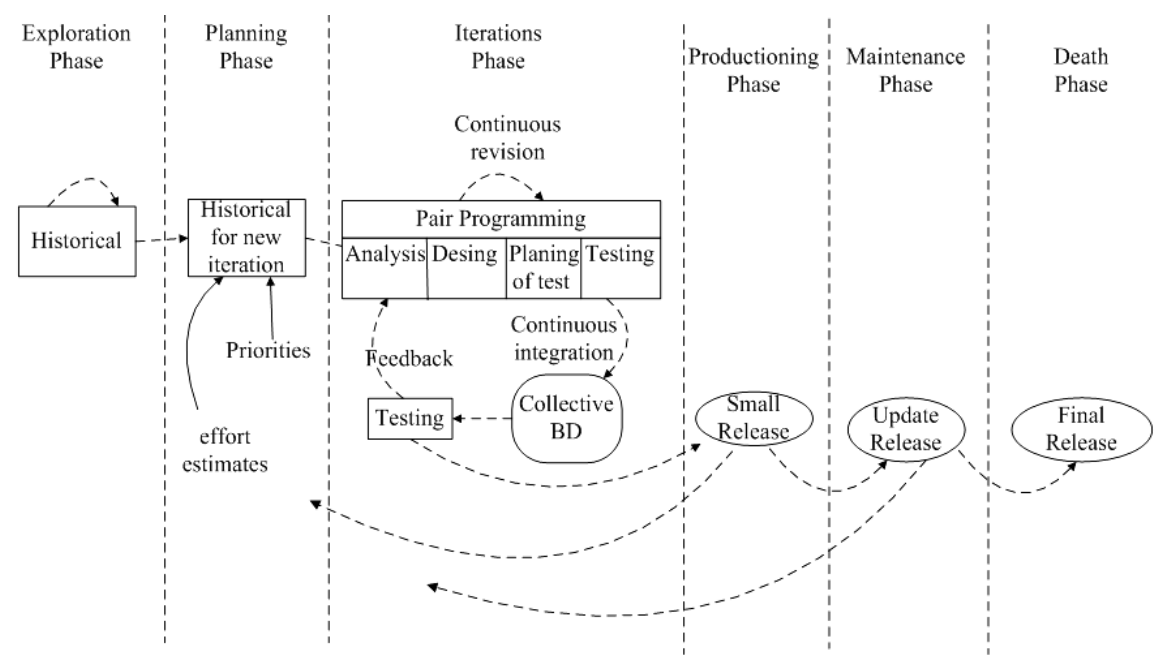

Figure 1 The eXtreme Programming Process.

The methodology is designed to deliver the software that customer needs when it's needed. This methodology emphasizes team work. Managers, customers, and 
developers are all part of a team dedicated to deliver quality soft-ware. XP implements a simple, yet effective way to enable groupware style development. XP improves a software project in four essential ways; communication, simplicity, feedback, and courage.

\subsection{The Roles in a XP Team}

XP defines the following roles for a software development process [8]:

- Programmer. The programmer writes source code for the software system under development. This role is at the technical heart of every XP project because it is responsible for the main outcome of the project: the application system.

- Customer. The customer writes user stories, which tell the programmer what to program. "The programmer knows how to program. The customer knows what to program".

- Tester. The tester is responsible for helping customers select and write functional tests. On the other side, the tester runs all the tests again and again to create an up-dated picture of the project state.

- Tracker. The tracker keeps track of all the numbers in a project. This role is familiar with the estimation reliability of the team. Whoever plays this role knows the facts and records of the project and should be able to tell the team whether they will finish the next iteration as planned.

- Coach. The coach is responsible for the development process as a whole. The coach notices when the team is getting "off track" and puts it "back on track." To do this, the coach must have experience with XP.

- Consultant. Whenever the XP team needs additional special knowledge, they "hire" a consultant who possesses this knowledge. The consultant transfers this knowledge to the team members, enabling them to solve the problem on their own.

- Big boss. The big boss or Manager provides the resources for the process. The big boss needs to have the general picture of the project, be familiar with the current project state, and know whether any interventions are needed to ensure the project's success.

\section{How the XP Creative Team Can Operate}

In relation with the structure dimension it's important to considerate how the team can operate. In order to implement the functionality of each role, we must considerate two aspects: basic organizational conditions and the pertinent creative process. 


\subsection{Basic Organizational Conditions}

The creative team performance is determined by the organizational conditions in which it's inserted [39, 31, 32, 40, 41]. Some conditions are necessary - although not sufficient - for the creative performance. We are interested in explore the influence of autonomy, communication, cooperation and learning, the handling of possible conflicts, pressure, formalization, performance evaluation, available resources (time) and the physical atmosphere of work.

The team member's communication, cooperation and learning are fortified since the client is present and there exist opened spaces to work together and in a pair programming mode. The work dynamics is based on a game of planning and metaphors involving all the participants from the beginning (client and equipment developer). Also, the use of codification standards, the small deliveries, the collective property of the code and the simple design, allow that the person has clear performance codes and rules about what is expected and acceptable (internal culture) in order to establish the required communication and cooperation.

The handling of possible conflicts between the client and the development team, and internally at team level is favored by XP practices handling it (presence of the client, pairs programming, planning game, continuous integration, tests, collective property), or to reduce it and to avoid it (small deliveries, simple design, 40 hour a week and codification standard). Cooperation and trust are associated to this issue.

The pressure (that in creativity is appraised as favorable until certain degree, favoring the performance, and detrimental if it exceeds this degree), is susceptible then to favor in XP through the client in situ, the programming by pairs, the planning game, the tests and continuous integration. It's possible to avoid, or at least to reduce, the pressure through the refactorization, the small deliveries, the collective property, and the fact that surpassing the 40 weekly working hours is seen like an error.

The formalization gives account of all those formal aspects (norms, procedures) defined explicitly and that are known, and even shared, by the members of the team. It's assured in XP through planning game, metaphors, continuous integration, the collective property, the 40 hours per week and the codification standards guiding the desirable conduct and performance of the team.

The evaluation of the performance is made in XP through pair programming (self evaluation and pair evaluation), frequent tests and even through the 40 weekly hours (as a metric indicating limit of effectiveness), all at the light of the planning (including the standards). Finally, the presence of client constitutes the permanent and fundamental performance evaluation of the team and the products. The evaluation characteristics empower the learning process.

The time dedicated has fundamental importance in XP team respecting the avail-able resources. This aspect is strongly stressed in creativity. The pair programming and the developer multifunctional role allow optimize the partial working-times, as well as the whole project time, ensuring a positive pressure. 
The physical atmosphere of work, referred in creativity to the surroundings that favor or make difficult the creative performance (including aspects like available spaces, noise, colors, ventilation, relaxation places) are assured only partially in $\mathrm{XP}$ through the open spaces, as a way to assure the interaction between members of the team.

\subsection{The Creative Process}

The team performance is directly determined by the creative process [31, 42]. It's important to correlate the phases defined in XP with the phases considered in a creative process.

The initial preparation and "finding" defined in the creative process correspond to the exploration phase in XP, where the functionality of the prototype and familiarization with the methodology are established.

The final stage of preparation is equivalent with the phases of exploration and planning in XP, defining more in detail the scope and limit of the development.

The option generation phases, incubation and election of options defined in the creative process correspond to the iterations made in XP and also with the liberations of the production phase (small releases). In XP there is not a clear distinction of the mentioned creative phases, assuming that they occur to the interior of the team.

The feedback phase (understanding this one as a final stage of the process, and not excluding that can have existed previous micro - feedbacks since the creative process is nonlinear) it could correspond in XP with the maintenance phase.

The persuasion phase is related to the phase of death established in XP, constituting the close of the development project with the final liberation.

\section{The Roles in Creativity and XP Roles}

Regarding to the structure dimension of a new product development team (in particular software), it is possible to relate the roles in creativity to the roles defined in the XP methodology distinguishing: base roles, that is, those directly related to the creative processes and software development; and support roles, whose function is to support or lead the other roles for a better performance. As previously mentioned in the creative process there are base and supporting roles. The base roles are directly related to the creative and software development process and the supporting roles support or lead the base roles to a better performance. The following is the correlation between creative and XP roles:

- The detective function consisting in collecting information related to a problem is made by the client himself in XP, because this one generates the first contact with the software development team. 
- The function of explorer consisting in defining completely the problem is made in XP as much by the client as the manager of the team, all together they appreciate the reach of the identified problem, as well as of the possible solutions. The function of the artist consisting in transforming the information, creating new relations, and therefore generating interesting solutions is made by the developer, that in XP methodology is in charge of the analysis, design and programming of software.

- The function of the engineer referred to clarify and to evaluate the new ideas, in terms of its feasibility is made in XP by the tester and the tracker.

- The function of the judge, understood as the definitive selection of the solutions to implant, is made in XP by the tracker and the client.

- The function of the producer, referred to the implementation of the selected ideas (strictly speaking it is working software) is made in XP by the client in his organization, including the processes and procedures that this function implies.

The supporting roles considered are:

- The provoker; creativity demands that the divergence as well as convergence in the solutions be maximum and complete. There is not explicit reference in XP methodology about divergent thinking.

- The think tank who helps the team work "from outside" is equivalent completely to the role of the consultant.

- The facilitator whose function is helping the team, corresponds in XP to the coach role.

- The manager whose function is to lead to the team in terms of its general efficiency and its effectiveness corresponds with XP's big boss or manager.

\section{Conclusions}

The Extreme Programming methodology includes implicitly central aspects of a creative teamwork. The structure that the team adopts and specially the different roles that the methodology advises to define, nearly correspond with the roles at the interior of a creative team. By other hand, the performance that characterizes the team through certain advisable practices, from the perspective of creativity, constitutes the necessary basic conditions, although non-sufficient, in order to favor the group creative performance. We think that XP methodology should have a more explicit reference to the provoker role that is thoroughly described in creativity as a fundamental factor to generate innovation. Furthermore, it is necessary a better distinction and formalization of the creative phases to generate options incubation and option choices (that are fundamental in creativity). It is 
assumed that they take place in the iterative and production process. Again, XP is not focused in "originality", resulting that the divergence is not so fundamental in XP. A more direct mention to the physical atmosphere of work, that in creativity are considered as highly relevant to enhance the performance, it is recommendable in XP.

\section{References}

1. Maurer J.M., Tessem F.: Human and social factors of software engineering: workshop summary. SIGSOFT Softw. Eng. Notes, 30(1-6) (2005).

2. Kotler P., Trías de Bes F.: Marketing Lateral, Editorial Pearson/Prentice Hall, Spain (2004).

3. Sutherland J.: Agile can scale: Inventing and reinventing scrum in five companies. Cutter IT Journal, 14, pp. 5-11 (2001).

4. Sutherland J.: Agile development: Lessons learned from the first scrum. Cutter Agile Project Management Advisory Service: Executive Update, 5, pp. 1-4 (2004).

5. Sutherland J.: Recipe for real time process improvement in healthcare. 13th Annual Physician Computer Connection Symposium, 2004. In Rancho Bernardo, CA: American Society for Medical Directors of Information Systems (AMDIS) (2004).

6. Sutherland J.: Future of scrurn: Parallel pipelining of sprints in complex projects. In: Agile, IEEE Computer Society, pp. 90-102 (2005).

7. Sutherland J., van den Heuvel W.J.: Towards an intelligent hospital environment: Adaptive workflow in the OR of the future. In: HICSS, IEEE Computer Society (2006).

8. Beck K.: Extreme programming explained: embrace change. Addison-Wesley Longman Publishing Co., Inc., Boston, MA, USA (2000).

9. Fruhling A.L., Tyser K., de Vreede G.J.: Experiences with extreme programming in telehealth: Developing and implementing a biosecurity health care application. In: HICSS, IEEE Computer Society (2005).

10. Christensen C., Bohmer R., Kenagy J.: Will disruptive innovations cure health care. Harvard Business Review pp. 102-111 (2000).

11. Dadam P., Reichert M., Kuhn K.: Clinical workflows - the killer application for process oriented information systems. BIS 2000 - Proceedings of the 4th International Conference on Business Information Systems pp. 36-59 (2000).

12. Fruhling A.: Examining the critical requirements, design approaches and evaluation methods for a public health emergency response system. Communications of the Association for Information Systems, 18 (2006).

13. Fruhling A.L., Steinhauser L., Hoff G., Dunbar C.: Designing and evaluating collaborative processes for requirements elicitation and validation. In: HICSS, IEEE Computer Society, 15 (2007).

14. Takeuchi H., Nonaka I.: The new product development game. Harvard Business Review (1986).

15. Gu M., Tong X.: Towards hypotheses on creativity in software development. Bomarius F., Iida H., eds., Computer Science, Springer vol. 3009, pp. 47-61 (2004).

16. Chau T., Maurer F., Melnik G.: Knowledge sharing: Agile methods vs tayloristic methods. Twelfth International Workshop on Enabling Technologies: Infrastructure for Collaborative Enterprises, WETICE, Los Alamitos, CA, USA, IEEE Computer Society, pp. 302-307 (2003).

17. Maiden N., Gizikis A., Robertson S.: Provoking creativity: Imagine what your requirements could be like. IEEE Software 21, pp. 68-75 (2004).

18. Glass R.L.: Software creativity. Prentice-Hall, Inc., Upper Saddle River, NJ, USA (1995). 
19. Crawford B., León de la Barra C.: Enhancing creativity in agile software teams. Concas, G., Damiani, E., Scotto, M., Succi, G. eds.: XP. vol. 4536, Computer Science, pp.161162 Springer (2007).

20. Robertson J.: Requirements analysts must also be inventors. Software, IEEE 22 pp. 48-50 (2005).

21. Maiden N., Robertson S.: Integrating creativity into requirements processes: Experiences with an air traffic management system. In: 13th IEEE International Conference on Requirements Engineering (RE 2005), 29 August - 2 September 2005, Paris, France, IEEE Computer Society, pp. 105-116 (2005).

22. Mich L., Anesi C., Berry D.M.: Applying a pragmatics-based creativity-fostering technique to requirements elicitation. Requirements Engineering, vol. 10, pp. 262-275 (2005).

23. Maiden N., Gizikis A.: Where do requirements come from? IEEE Softw. 18, pp. 10-12 (2001).

24. Robertson J.: Eureka! why analysts should invent requirements. IEEE Softw. 19, pp. 20-22 (2002).

25. Boden M.: The Creative Mind, Abacus (1990).

26. Memmel T., Reiterer H., Holzinger A.: Agile methods and visual specification in software development: a chance to ensure universal access. In: Coping with Diversity in Universal Access, Research and Development Methods in Universal Access. Computer Science, vol. 4554, pp. 453462 (2007).

27. Holzinger A.: Rapid prototyping for a virtual medical campus interface. IEEE Software 21, pp. 92-99 (2004).

28. Holzinger A., Errath M.: Designing web-applications for mobile computers: Experiences with applications to medicine. In Stary C., Stephanidis C., eds, User Interfaces for All. Computer Science, vol. 3196, pp. 262-267 Springer (2004).

29. Holzinger A., Errath M.: Mobile computer web-application design in medicine: Research based guidelines. In: Springer Universal Access in Information Society (in print) (2007).

30. Amabile T., Conti R., Coon H., Lazenby J., Herron M.: Assessing the work environment for creativity. Academy of Management Journal, vol. 39, pp. 1154-1184.

31. Leonard D.A., Swap W.C.: When Sparks Fly: Igniting Creativity in Groups. Harvard Business School Press, Boston (1999).

32. Woodman R.W., Sawyer J.E., Griffin R.W.: Toward a theory of organizational creativity. The Academy of Management Review, vol. 18, pp. 293-321 (1993).

33. Welsh G.: Personality and Creativity: A Study of Talented High School Students. Unpub. doctoral dissertation, Chapel Hill, University of North Carolina (1967).

34. Csikszentmihalyi M.: Creativity: Flow and the Psychology of Discovery and Invention, Harper Perennial, New York (1998).

35. Guilford J.P.: Intelligence, Creativity and Their Educational Implications. Edits Pub (1968).

36. Hallman R.: The necessary and sufficient conditions of creativity. Journal of Humanistic Psychology, vol. 3 (1963) Also reprinted in: Gowan J.C. et al., Creativity: Its Educational Implications. John Wiley and Co., New York (1967).

37. Hallman R.: Aesthetic pleasure and the creative process. Journal of Humanistic Psychology, 6, pp. 141-148 (1966).

38. Hallman R.: Techniques of creative teaching. Journal of Creative Behavior, vol. I (1966).

39. Amabile T.: How to kill creativity. Harvard Business Review, pp. 77-87, Sept-Oct (1998).

40. Kotler P., Armstrong G.: Principles of Marketing, 10th Edition, Prentice Hall (2003).

41. Isaksen S.G., Lauer K.J., Ekvall G.: Situational outlook questionnaire: A measure of the climate for creativity and change. Psychological Reports, pp. 665-674.

42. Wallas G.: The art of thought, Harcourt Brace, New York (1926).

43. Lumsdaine E., Lumsdaine M.: Creative Problem Solving: Thinking Skills for a Changing World. McGraw-Hill, Inc, New York (1995).

44. Kelley T., Littman J.: The Ten Faces of Innovation: IDEO's Strategies for Defeating the Devil's Advocate and Driving Creativity Throughout Your Organization. Currency (2005). 